\title{
Usefulness of a Vascular Clipping System to Create an Arteriovenous Fistula
}

\author{
Jin Suk Lee a, $\uparrow$, Kuk-Jin Nam b, $\dagger$, Sam-Youl Yoon c, Kun Ok Lee d, Hyung Joon Han d, Sung-Jin Cho e, \\ Jong-Woong Park ${ }^{\mathrm{f}}$, Tae Jin Song d,* \\ a Department of Trauma Surgery, Chungbuk National University Hospital, Cheongju, Korea \\ ${ }^{b}$ Department of Otorhinolaryngology, Korea University Hospital, Ansan, Korea \\ ${ }^{c}$ Department of Surgery, Hallym University, Seoul, Korea \\ ${ }^{d}$ Department of Surgery, Korea University Hospital, Ansan, Korea \\ ${ }^{e}$ Department of Pathology, Hallym University, Seoul, Korea

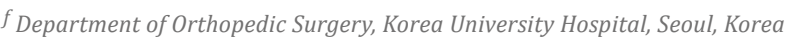

\section{Article history:}

Received: August 11, 2019

Revised: March 2, 2020

Accepted: March 5, 2020

\author{
$\dagger$ These authors contributed equally to \\ this study. \\ *Corresponding Author: \\ Tae Jin Song \\ Department of Surgery, Korea University, \\ Ansan, 15355, Korea. \\ E-mail: lesilles@gmail.com \\ ORCID \\ Jin Suk Lee \\ https://orcid.org/0000-0002-6694-4683 \\ Kuk Jin Nam \\ https://orcid.org/0000-0002-8128-3107 \\ Kun Ok Lee \\ https://orcid.org/0000-0001-9419-3605 \\ Hyung Joon Han \\ https://orcid.org/0000-0002-7830-5352 \\ Tae Jin Song \\ https://orcid.org/0000-0001-9278-812X
}

\section{ABSTRACT}

Purpose: The vascular clipping system (VCS) is beneficial as it is simple and easy to apply for microvascular suturing. Arteriovenous fistula (AVF) creation is a very basic standard technique of microvascular surgery. In this study the VCS and the conventional suture methods were compared in a rabbit model using the carotid artery and vein to create an AVF.

Methods: There were 28 rabbits assigned equally into 2 groups using the AVF creation method (conventional suturing or the VCS procedure). Histopathology was performed on fixed samples. The procedure time of the 2 methods and changes in histopathology of tissue samples after surgery were compared.

Results: The VCS procedure showed a lower degree of fibrosis and hyperplasia histologically compared with the conventional suture method. The VCS was quicker to perform and no significant anastomosis stricture was observed.

Conclusion: In a rabbit model of AVF, the VCS has benefits over the conventional suture method. The VCS provides comparable patency rates, produces fewer side effects such as fibrosis and hyperplasia, and takes less operation time than suturing. The VCS is expected to be useful for cases where renal patients need periodic hemodialysis and thus repetitive access to a vessel.

Keywords: arteriovenous fistula.

\section{Introduction}

In 1992, Kirsch et al [1] developed a vascular clipping system (VCS) model. The VCS was devised to implement vascular anastomosis with ease. It also takes less time in surgery alleviating pressure on the surgeon. In addition, the VCS has a better prognosis than the conventional suturing anastomosis system. These benefits have been shown experimentally, however, the clinical application of VCS is restricted until its safety and efficacy can be shown [2].

Treating kidney disease is crucial for the quality of life in patients with chronic kidney disease because if left untreated it can lead to various complications such as diabetes mellitus, azotemia, and hypertension. As the patients undergo repeated hemodialysis, long-term vascular access matters, and arteriovenous fistulas (AVF) are considered the best form for hemodialysis.

As a vein meets an artery in an artificial manner, it becomes 
more stable and thicker in pathology, which makes repetitive hemodialysis feasible. The AVF uses a rigid Teflon tube which was developed by Quinton in 1960. The conventional operational method linking a radial artery and a cephalic vein was developed by Brescia in 1966 [3]. This vascular clipping system can be used to make the AVF for chronic kidney disease patients. It is anticipated that the AVF made by vascular clipping will show a higher patency rate, which is useful in dialysis in the long term. With this VCS technique, intimal hyperplasia of associated vessels can be minimized and the incidence of obstructive problems should decrease. This is partly because the VCS does not completely penetrate the intimal layer of the blood vessels [2].

In order to substantiate these facts, preliminary experimental data is necessary in an animal model to compare between the typical suturing system and the VCS. The advantage of the VCS over the conventional suture method in clinical application can be reviewed by statistical analysis. Histological change of the VCS tissue in humans is hard to carry out, therefore, histological analysis has previously been performed on a narrow range and depth of tissue. Moreover, histological analysis on resected tissue where the VCS has been applied, has never been performed.

Another enormous advantage of the VCS is the simplicity in learning the surgical technique. The mechanism of the VCS is quite straightforward in that it enables many surgeons who have not learnt specialized microsurgery skills to become an expert at an anastomosis technique.

This study examines the VCS in an animal model and the associated results. Specimens from before and after the VCS were taken and histopathology was performed and comparison with the conventional suture technique analyzed statistically.

\section{Materials and Methods}

There were 28 randomly selected New Zealand rabbits, weighing between $2.5 \mathrm{~kg}$ and $3.5 \mathrm{~kg}$, used for this study in accordance with the Korean University animal care criteria. In order to acclimatize these animals, they were housed under the same standard circumference and condition according to the guidelines until tissue sample collection. These animals were classified into 2 groups, categorized by the operation methods.

After they were treated with pentobarbital $25 \mathrm{mg} / \mathrm{kg}$ and diclopenac- $\beta$ diethylaminoethanol $1.5 \mathrm{mg} / \mathrm{kg}$, as an anesthetic during surgery, the rabbits were fastened in a supine position on an operating table. The cervical area was shaved and left jugular vein and artery were dissected about $2.5 \mathrm{~cm}$ to $3 \mathrm{~cm}$. After the vessels were fixed using an Acland double microvascular clamp, they were opened by an incising scalpel.

In the control group, rabbits were operated on using conventional suture techniques. After opening the specific region, the repairing process was performed in continuous side to side anastomosis using 9-0 monofilament nylon. Rabbits in the experimental group were operated on by fixing the anastomosis at the end of the vessel using 8-0 monofilament nylon and were anastomosed by using the VCS (VCS clip, Vascular Closure Staple, Unites States Surgical Corporation, Norwalk, CT; Figure 1). All procedures were performed by 1 operator. Every anastomosis was $12 \mathrm{~mm}$ in size.

Three months after the initial operation, the rabbits were anesthetized as described previously and the anastomotic sites (both venous and arterial side) were cut out approximately measuring $12 \mathrm{~mm}$ in length. The animals were sacrificed after tissue sampling.

After the tissues were washed with PBS solution, they were immersed in glutaraldehyde for 24 hours. The samples were embedded in paraffin and stained with hematoxylin and eosin. Three slide sections for each rabbit were examined under the microscope $(\times 40, \times 100, \times 200)$ and 3 sample pictures were taken to determine intimal growth and hyperplasia. Scanning microscopy was used to examine intimal tissue formation, and intimal hyperplasia at the anastomotic sites.

Masson's Trichrome stain was used to detect fibrotic tissue. Anastomotic sections were hydrated in distilled water. Hydrated tissues were processed with Bouin's solution and then heated in a microwave oven for 1 minute. In order to remove the picric acid, the samples were washed with tap water for 10 minutes. The tissue samples were stained with Weigert's working solution for 10 minutes and then rinsed in warm tap water for 10 minutes. After that, Biebrich scarlet-acid fuchsin solution was added and left to stand for 10 minutes, then tissues were washed with distilled water.

For differentiation of the tissue sections, phosphomolybdic/ phosphotungstic acid solution was added for 10 minutes until it turned pale pink. Sections were then submerged in aniline blue solution without rinsing, and left to stain for 10 minutes, then rinsed with distilled water, and differentiated in $1 \%$

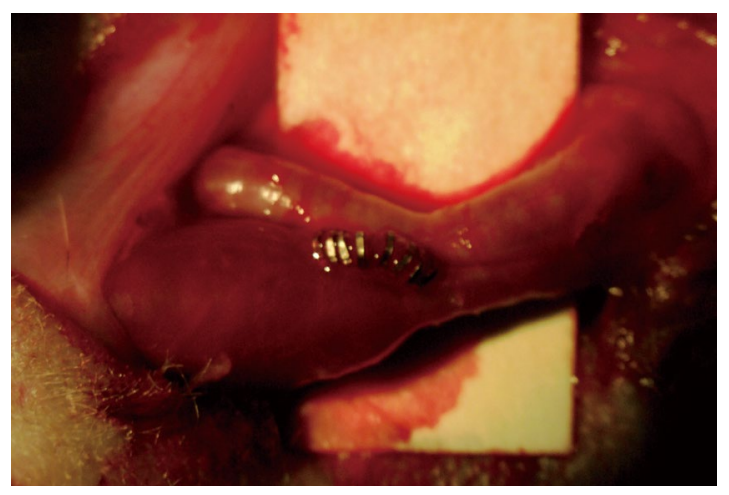

Figure 1. Anastomosis was performed at $0.5 \mathrm{~mm}$ intervals using small sized titanium clips. 


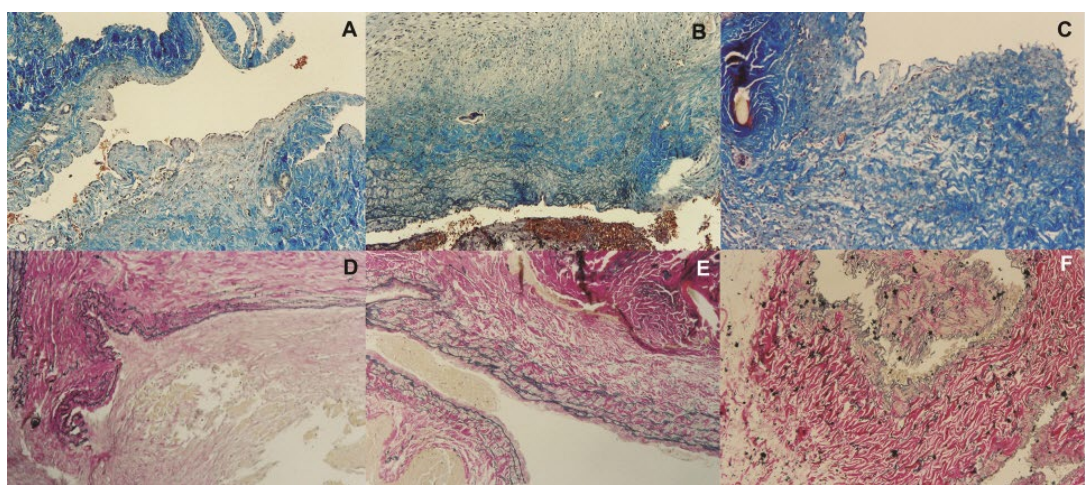

Figure 2. Masson's Trichrome stain showed the degree of tissue fibrosis. Tissue fibrosis grade was from 1 to 3: (A) Grade 1, (B) Grade 2, (C) Grade 3. Elastic Van Gieson staining was used to evaluate the degree of elastic tissue hyperplasia. The pathologist determined the degree of elastic tissue hyperplasia from 0 to 2: (D) Grade 0, (E) Grade 1, (F) Grade 2.

acetic acid solution for 5 minutes. As a final step, the tissues were dehydrated with 95\% ethyl alcohol. Dehydrated tissues were mounted with a mounting medium. One pathologist determined the tissue fibrosis grade of the section (Figures 2AC).

Elastic Van Gieson stain was used especially to evaluate the degree of elastic tissue hyperplasia. Hydrated anastomotic segments were oxidized in potassium permanganate for 5 minutes and rinsed in distilled water. Bleaching by oxalic acid was performed for 5 minutes and was washed out. All tissue samples were rinsed in $95 \%$ ethanol and stained in Elastic stain for 20 hours. After that, they were thoroughly rinsed in distilled water and counterstained with Elastic Van Gieson stain solution for 1 minute. Processed sections were dehydrated in $100 \%$ ethanol and xylene and mount with mounting media. The pathologist determined the degree of elastic tissue hyperplasia (Figures 2D-F).
Statistical analysis was performed using SPSS, Version 12.0 for Windows (SPSS Inc., Chicago, IL, USA). Comparisons between groups were tested using the independent $t$ test, and $p<0.05$ were considered statistically significant.

\section{Results}

The mean degree of AVF fibrosis graded in the conventional suture control group $(2.1 \pm 0.7)$ was higher than the mean degree of fibrosis in the VCS experimental group $(1.0 \pm 0.0$; Table 1$)$. This difference was statistically significant $(p=0.013)$ and was observed immunohistopathologically (Figures $3 \mathrm{~A}$ and 3B).

The mean degree of elastic tissue hyperplasia was $1.6 \pm 0.5$ graded in the conventional suture control group. There was
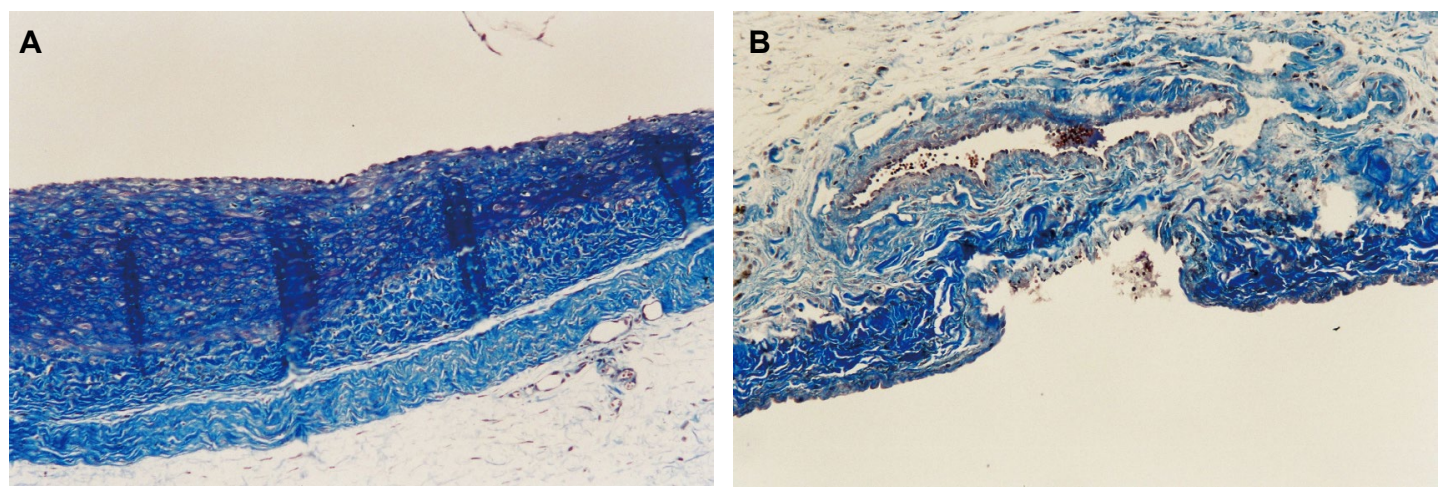

Figure 3. A comparison of arteriovenous fistula fibrosis was performed between (A) the vascular clipping system experimental group and (B) conventional suture control group. No direct injury of the intima layer was detected. 

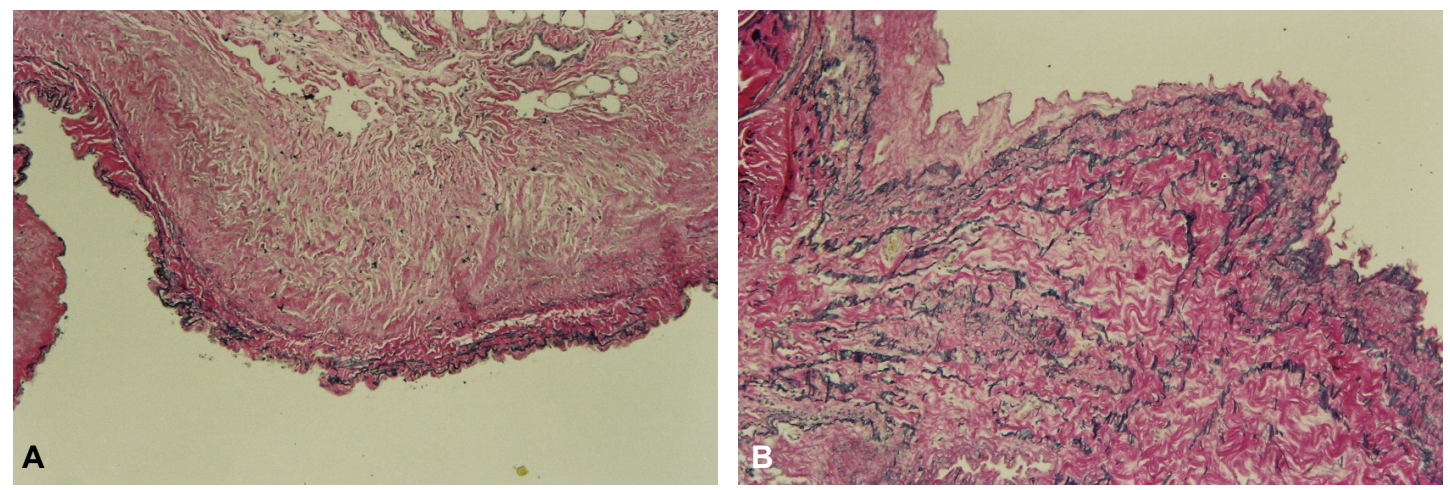

Figure 4. The degree of elastomeric hyperplasia in the vessel clipping system experimental group (A) was significantly lower than the conventional suture control group (B).

no hyperplasia detected in the VCS experimental group. This difference between groups was statistically significant ( $p=$ 0.001 ) and was observed immunohistopathologically (Figures $4 \mathrm{~A}$ and $4 \mathrm{~B})$.

The arterial wall of AVF in VCS group was thicker than the conventional suturing group ( $2.9 \pm 0.4$ vs. $2.6 \pm 0.4)$, and the venous wall of the AVF in the VCS group was thicker than the conventional suture group $(2.7 \pm 0.5$ vs. $2.8 \pm 0.6)$. However, there was no statistically significant difference detected between groups for the arterial ( $p=0.456$ ) and venous wall ( $p$ $=0.710$ ) thicknesses of the AVF.

The mean anastomotic operation time in the VCS group was statistically significantly shorter than the control group [13.8 \pm 3.8 vs $19.0 \pm 3.6$ minutes $(p=0.026)$; Table 1$]$.

\section{Discussion}

In every area in the medical field, vessel suturing contributes to vascular repair and anastomosis. Since the first attempt to link 2 vessels by sewing, the anastomosis technique has observed dramatic developments. However, the most widely used hand-sewn suturing technique has many complications such as stenosis, intimal hyperplasia, intimal dissection, and thrombosis. Moreover, it is difficult to master the fundamentals of the vascular anastomosis technique. It also required time and experience to gain expertise in microvascular anastomosis techniques. Through continued effort to reduce these critical complications, and to facilitate the technique, Kirsch et al [1] developed the VCS for anastomosis in 1992.

In this study the conventional suturing method was compared with the VCS technique for AVF, and a significantly shorter operation time for vessel anastomosis was observed using the VCS than using the conventional suture technique. Pathological analysis showed less fibrosis in the anastomosis site and less elastic tissue formation in the intimal layer in the VCS group compared with the conventional suture group.

Intimal hyperplasia and fibrosis of the anastomosis may cause poor access and occlusion by creating an AVF. Intimal hyperplasia was first described by Carrel and Guthrie in 1906. Anastomosis was covered with a reluctant material for a few days after a vascular operation. In current practice, anastomosis during bypass surgery uses prosthetic bypass

Table 1. Comparison of fibrosis, elastic tissue hyperplasia, vessel thickness, and operative time between the vascular clipping system and conventional suture control group.

\begin{tabular}{lccc}
\hline & VCS $(n=14)$ & Suture $(n=14)$ & $p$ \\
\hline Degree of fibrosis (Grade 1-3) & $1.0 \pm 0.0$ & $2.1 \pm 0.7$ & $0.013^{*}$ \\
Degree of hyperplasia (Grade 0-2) & 0 & $1.6 \pm 0.5$ & $0.001^{*}$ \\
Artery size (mm) & 2.92 & 2.64 & 0.456 \\
Vein size (mm) & 2.83 & 2.79 & 0.71 \\
Operation time (min) & 13.25 & 19 & $0.026^{*}$ \\
\hline
\end{tabular}

* Statistically significant.

VCS = vascular clipping system. 
grafts, denuded arteries, or arterialized vein [4].

Injury to the vascular endothelium stimulates a series of events that results in the transformation of a subset of smooth muscle cells initiating proliferation and migration from the media to the intima. The degree of intimal hyperplasia may be evaluated histologically by the degree of formation of elastic tissue [4]. In this study, direct injury of the intima layer and elastic tissue formation were not observed histologically (Figure 3A). Intimal approximation technique by VCS resulted in no elastic tissue formation and no intimal hyperplasia in the VCS group. The levels of intimal endothelial hyperplasia, macrophage and leukocyte infiltration, and endothelial wall fibrosis were significantly reduced. The round-ended design of the clips result in a pathologically favorable vascular status. Microvascular reconstruction using the VCS based on the principle of non-penetrating, curved intimal approximation by an arcuate angled clip has proven histologically superior to the penetrating conventional suture technique.

In the other studies, it has also been suggested that the non-penetrating features of the VCS could reduce risk factors such as intimal hyperplasia, operative intimal injury, mural ischemia, and platelet deposition. The absence of direct intimal injury and foreign bodies could result in an intact intimal layer and higher patency rate [5].

The operating time is consistently shorter with the VCS, compared with the conventional suturing control group. This time saving benefit of the VCS has also been described as a form of vascular surgery taking less time and with less bleeding $[2,3,6]$. As a result, the VCS decreases the required time for surgical operation. Usually, fatigue and stress for the transplant surgeon is related to the operation time. Therefore, prolonged operation time often produces poor results in transplant surgeries $[7,8]$. The clinical use of the VCS could result in a reduction of the total operation time.

Another study showed different degrees of anastomosis fibrosis depending on the materials used. Silver clips cause more anastomosis fibrosis than stainless steel clips, even though they are similar in shape and function (such as everting and non-penetrating). [9] Thus, new materials could be considered such as a co-polymer of polylactic acid or polyglycolic acid, which are components of the subcutaneous stapler.

This study was performed in small animals with relatively lower blood pressure and faster blood flow than humans. In humans, tension and rough handling performed during vascular anastomosis for transplantation or joint surgery could result in intimal injury and dissection. Nevertheless, this vascular anastomosis technique is useful in various fields of surgery. The beneficial results of vascular anastomosis depend on the surgeon's skill and experience. Less time and effort is necessary for mastering the standardized VCS technique and using an arcuately legged clip has the potential to apply this method to various clinical fields, such as the repair of transected nerve [10], vascular surgery, kidney transplantation, cardiothoracic surgery [11], and liver transplantation [12]. This method is highly effective with medical merit. However, because of the small sample size in this animal model study, this data should be viewed cautiously before clinical use. The safety and security of the VCS requires further evaluation on a larger scale.

\section{Conflicts of Interest}

There were no potential conflicts of interest to report.

\section{References}

[1] Kirsch WM, Zhu YH, Hardesty RA, Chapolini R. A new method for microvascular anastomosis: Report of experimental and clinical research. Am Surg 1992:58(12):722-7.

[2] Baguneid MS, Goldner S, Fulford PE, Hamilton G, Walker MG, Seifalian AM. A comparison of para-anastomotic compliance profiles after vascular anastomosis: nonpenetrating clips versus standard sutures. J Vasc Surg $2001 ; 33(4): 812-20$.

[3] Yu HC, Lee TH, Jeong YJ, Cho BH. Vascular Clips in the Creation of Arteriovenous Fistula for Hemodialysis.

[4] Davies MG, Hagen PO. Pathobiology of intimal hyperplasia. Br J Surg 1994;81(9):1254-69.

[5] Park JW, Kim AR, Kim SK, Park JH, Hong JS, Shin JH. Microvascular Anastomosis of Rabbit\&apos;s Jugular Vein with Titanium Clip. J Korean Orthop Assoc 2002;37(2):269-73.

[6] Leppäniemi A, Wherry D, Pikoulis E, Hufnagel H, Waasdorp C, Fishback $\mathrm{N}$, el al. Arterial, and venous repair with vascular clips: Comparison with suture closure. J Vasc Surg 1997;26(1):24-8.

[7] Kaufman HH, Wiegand RL, Tunick RH. Teaching surgeons to operatePrinciples of psychomotor skills training. Acta Neurochir (Wien) 1987;87(1-2):1-7.

[8] Samkoff JS, Jacques $\mathrm{CH}$. A review of studies concerning effects of sleep deprivation and fatigue on residents\&apos; performance. Acad Med 1991;66(11):687-93.

[9] Samuels PB. Method of Blood Vessel Anastomosis by Means of Metal Clips: An Experimental Study. AMA Arch Surg 1955;70(1):29-38.

[10] Okui N, Yamamoto M, Fukuhira Y, Kaneko H, Hirata H. A new nerve coaptation technique using a biodegradable honeycomb-patterned film. Microsurgery 2012;32(6):466-74.

[11] Boeckxstaens GE, Pelckmans PA. Nitric oxide and the non-adrenergic non-cholinergic neurotransmission. Comp Biochem Physiol A Physiol 1997;118(4):925-37.

[12] Geevarghese SK, Bradley AL, Atkinson J, Wright JK, Chapman WC, Van Buren DH, et al. Comparison of arcuate-legged clipped versus sutured hepatic artery, portal vein, and bile duct anastomoses. Am Surg 1999;65(4):311-6. 